\title{
Redox signaling regulates commensal-mediated mucosal homeostasis and restitution and requires formyl peptide receptor 1
}

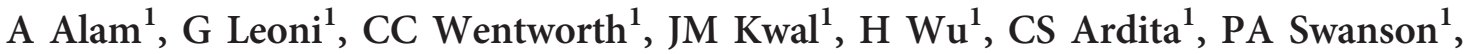 \\ JD Lambeth ${ }^{1}$, RM Jones ${ }^{1}$, A Nusrat ${ }^{1}$ and AS Neish ${ }^{1}$
}

The mammalian gut microbiota is essential for normal intestinal development, renewal, and repair. Injury to the intestinal mucosa can occur with infection, surgical trauma, and in idiopathic inflammatory bowel disease. Repair of mucosal injury, termed restitution, as well as restoration of intestinal homeostasis involves induced and coordinated proliferation and migration of intestinal epithelial cells. $\boldsymbol{N}$-formyl peptide receptors (FPRs) are widely expressed pattern recognition receptors that can specifically bind and induce responses to host-derived and bacterial peptides and small molecules. Here we report that specific members of the gut microbiota stimulate FPR1 on intestinal epithelial cells to generate reactive oxygen species via enterocyte NADPH oxidase 1 (NOX1), causing rapid phosphorylation of focal adhesion kinase (FAK) and extracellular signal-regulated kinase mitogen-activated protein kinase. These events stimulate migration and proliferation of enterocytes adjacent to colonic wounds. Taken together, these findings identify a novel role of FPR1 as pattern recognition receptors for perceiving the enteric microbiota that promotes repair of mucosal wounds via generation of reactive oxygen species from the enterocyte NOX1.

\section{INTRODUCTION}

The gastrointestinal epithelium acts as a physiologic barrier that separates the underlying systemic tissue compartments from the microbe-rich gut luminal contents. Defects in this barrier occur with inflammatory bowel diseases as well as infectious and mechanical injury. ${ }^{1,2}$ In addition, intrinsic defects in mucosal barrier homeostasis are likely central to the pathogenesis of inflammatory bowel diseases and systemic inflammatory syndromes. The mammalian intestinal tract is host to, and coevolved with, a taxonomically diverse and numerically vast prokaryotic microbiota. The resident microbial community in the intestinal lumen is essential for numerous beneficial functions including nutrient extraction, immune development, and competitive exclusion of pathogens. ${ }^{3}$ In addition, recent data have implicated the microbiota in the mucosal tissue development and renewal. Germ-free mice exhibit slower turnover of the intestinal epithelial cells $\left(\right.$ IECs) ${ }^{3}$ and epithelial regenerative responses to mucosal injury are impaired, suggesting a role for the microbiota in stimulation of wound restitution and response to injury. ${ }^{4-6}$ However, the mechanistic processes by which the host perceives the enteric microbiota to stimulate these beneficial effects is largely unknown.

The formyl peptide receptors (FPRs) are G-protein-coupled transmembrane receptors originally characterized in phagocytes, which upon stimulation by microbially derived formylated peptides, induce chemotaxis and reactive oxygen species (ROS) generation in these cells, ${ }^{7-10}$ indicating the role of FPRs as pattern recognition receptors. Functional FPRs are described in the IECs that serve as receptors for endogenous anti-inflammatory and proresolution mediators, including annexin A1 (AnxA1), lipoxin A4, and resolving D1. ${ }^{11-14}$ Recently, Chen et al. ${ }^{15}$ utilized FPR2-null mice to demonstrate defective healing in models of chemical colits, suggesting functional roles of the FPRs in the intestinal mucosa. Importantly, our prior work showed that AnxA1 requires FPR1 and the enterocyte NADPH oxidase, NADPH oxidase 1 (NOX1), for mucosal wound healing ${ }^{13}$ in a ROS-dependent

${ }^{1}$ Epithelial Pathobiology and Mucosal Inflammation Research Unit, Department of Pathology and Laboratory Medicine, Emory University School of Medicine, Atlanta, Georgia, USA. Correspondence: A Nusrat (anusrat@emory.edu) or AS Neish (aneish@emory.edu)

Received 27 May 2013; accepted 9 September 2013; published online 6 November 2013. doi:10.1038/mi.2013.84 
manner. Physiological levels of cellular ROS function as a signaling molecule and modulate different homeostatic processes, including those implicated in migration and cellular proliferation. ${ }^{16-18}$ FPRs expressed in IECs are in direct contact with the gut luminal contents ${ }^{11}$ and thus can also be activated by exogenous ligands, including microbial formyl peptides and certain taxa of the gut microbiota. ${ }^{15,19}$

We and others reported that specific taxa of enteric bacteria, largely lactobacilli, stimulate ROS production in the gut of animals as disparate as mammals and flies. ${ }^{13,20,21}$ (Jones et al., unpublished data). Recently, we demonstrated that microbiotainduced ROS generation promotes migration of cultured enterocytes by activating phosphorylation of focal adhesion kinase (FAK) in a redox-dependent manner. ${ }^{21}$ FAK is a key regulatory protein of focal adhesion complex that mediates turnover of cell adhesions, affects cell migration, and thus regulates wound repair. ${ }^{22,23}$ However, the receptor that recognizes bacterial signals and the cellular source of ROS that promote enterocyte migration, and thus mediate microbiota-induced intestinal wound healing and homeostasis, are unknown.

In this report, we examined the role of FPR1 as pattern recognition receptors in perception of enteric commensalmediated signaling in vivo and demonstrated that FPR1 mediates commensal bacteria-stimulated NOX1-dependent ROS generation in colonic enterocytes, which in turn activates phosphorylation of FAK and extracellular signal-regulated kinase (ERK) in vivo, culminating in migration and proliferation that are required for intestinal homeostasis and wound restitution. Overall, these results identify redox signaling as a key mechanism in the host crosstalk of the microbiota in homeostatic and restitutive processes.

\section{RESULTS}

\section{Enteric commensal bacteria-stimulated colonic mucosal wound healing requires FPR1 and NOX1}

To assess the extent to which FPR1 and NOX1 function in microbiota-induced restitution, discrete, and reproducible mechanical wounds (Supplementary Figure S1 online and Figure 1a) were inflicted in murine colonic epithelial mucosa with veterinary endoscopic biopsy forceps, followed by intrarectal administration of control (Hank's buffer; Hank's balanced salt solution (HBSS)) or Lactobacillus rhamnosus GG (LGG), a representative commensal bacterium that activates FPRs in cultured IEC. ${ }^{19}$ LGG-treated wound beds rapidly re-epithelialized within 2 days, whereas HBSS treated wounds re-epithelialized at a significantly slower rate demonstrated by the monolayer of $\beta$-catenin-containing epithelial cells (green) covering the wound beds (Figure $\mathbf{1 b}$ and Supplementary Figure S1c). LGG-enhanced wound closure was quantified utilizing the method described by Seno et al. ${ }^{24}$ in wild-type (WT) mice, in mice with germline mutations in FPR1 (Fpr1 ${ }^{-1}$ $\left.{ }^{-}\right)$, and in mice harboring an epithelial specific knockout in Nox1 $\left(\mathrm{Nox}^{\mathrm{f} / \mathrm{FL}} \times\right.$ Villin-Cre, designated $\left.\mathrm{Nox}^{-/-I E C}\right)$ (Supplementary Figure S1d and Figure 1c,d). In WT mice, wounds treated with LGG for 6 days showed enhanced mucosal closure (78.5\%) when compared with control treatment (57.4\%). However, LGG failed to enhance wound closure in Fpr1 ${ }^{-1-}$ and Nox1 ${ }^{-1-I E C}$ mice (59.3\% and $62.7 \%$, respectively) (Figure 1c,d). Untreated $\mathrm{Fprl}^{-1-}$ and Nox $1^{-/-I E C}$ mice did not show significant delays $(48.0 \%$ and $53.1 \%$, respectively) in wound healing when compared with WT mice. These data demonstrate that commensal bacteria augment repair of mucosal injury that requires FPR1 and NOX1 signaling pathways. In a separate model of epithelial restitution, mice were given $4.0 \%$ dextran sodium sulfate (DSS) in drinking water and disease activity index (body weight loss, stool consistency, and occult blood) was recorded. DSS was withdrawn and replaced with water, and animals were subsequently treated with intrarectal administration of LGG suspension or control for 3 days. As reported previously, ${ }^{21}$ LGG treatment (red) enhanced recovery from DSS-induced injury (Figure 1e). Importantly, the beneficial effects of LGG after DSS treatment were absent in Fpr1 ${ }^{-/-}$mice (violet). Collectively, these data demonstrate that FPR1 is also necessary in microbiota-induced recovery from intestinal injury induced by DSS treatment.

\section{Enteric commensal bacteria induce FPR1- and NOX1- dependent ROS generation in murine colonic epithelia} We hypothesized that, similar to AnxA1, products of the resident microbiota recognized by FPR1 require epithelial NOX1 to generate ROS and activate signaling pathways that eventuate in epithelial migration and restitution. Therefore, we investigated the extent to which commensal bacteria or fMetLeu-Phe (fMLF) activated FPRs situated on the apical surface of epithelial cells and mediated ROS response. Cellular ROS was determined in cultured polarized SK-CO15 colonic epithelial cells using redox-sensitive CM- $\mathrm{H}_{2}$ DCF-DA dye. We demonstrated that both LGG and AMLF stimulated ROS generation within $15 \mathrm{~min}$ in cultured SK-CO15 epithelial cells in a dosedependent manner (Supplementary Figure S2a), highlighting the enterocytes' ability to generate independently cellular ROS following bacterial contact. In addition, these ROS were produced in the cytoplasmic area of the epithelial cells but not in the nucleus (Supplementary Figure S2a, right panel). Next, we examined effects of PMLF and LGG to induce ROS generation in wounded model polarized epithelial cells. ROS were rapidly generated in cells within $15 \mathrm{~min}$ of application with fMLF and LGG (Figure 2a) Intriguingly, in response to application of fMLF and LGG, cells located in close proximity of the wound edges produced more ROS evident from the stronger fluorescent signals generated from CM$\mathrm{H}_{2}$ DCF-DA. In addition, LGG-induced ROS generation in wounded cultured enterocytes was abrogated in cells pretreated with Boc2, a specific and competitive inhibitor of fMLF binding to FPRs, and also by $\mathrm{N}$-acetyl-cysteine (NAC), a glutathione precursor and ROS scavenger (Figure 2a). These data demonstrate that LGG activated FPR to induce ROS generation in the wounded model polarized epithelial cells.

We next investigated whether commensal bacteria-induced ROS generation occurs in vivo in biopsy-wounded intestinal 
a

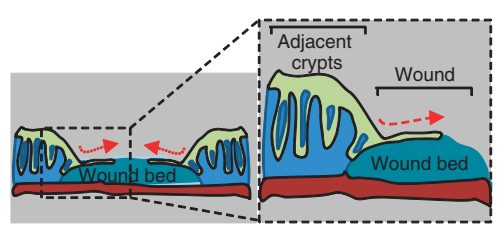

$\square$ Epithelium $\square$ Lamina propia $\square$ Muscularis propia
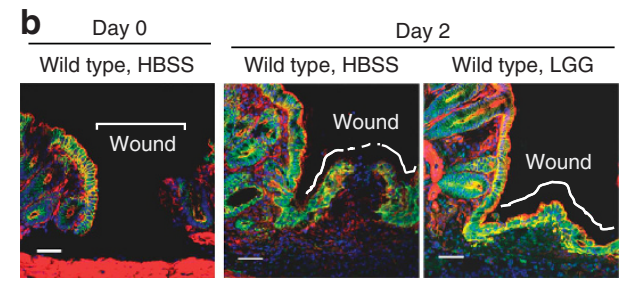

$\beta$-Catenin Actin DNA
C

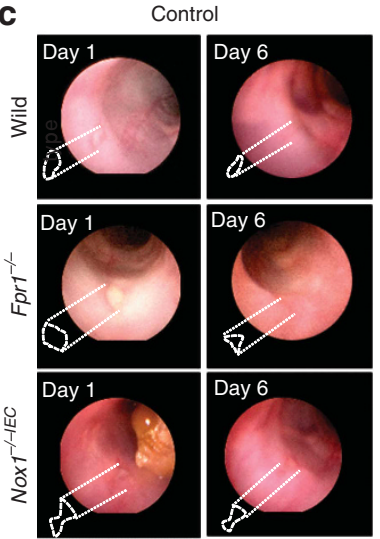

L. rhamnosus GG Treated

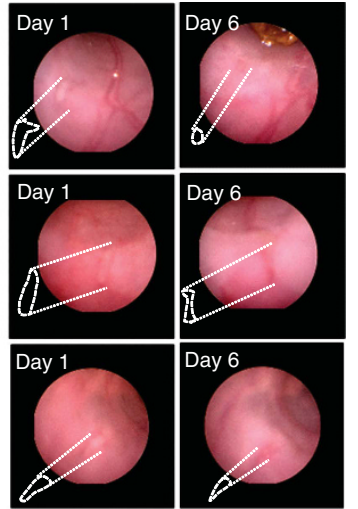

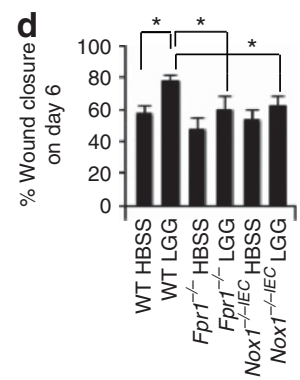

e

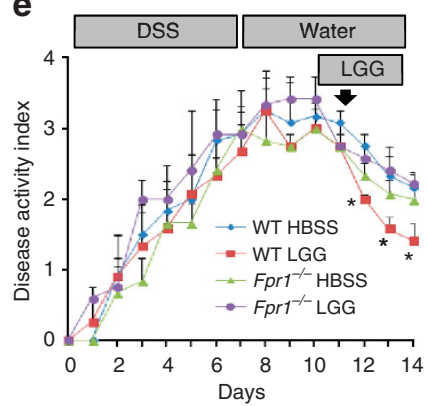

Figure 1 Enteric commensal bacteria require formyl peptide receptor 1 (FPR1) and NADPH oxidase 1 (NOX1) to enhance colon mucosal healing. (a) Schematic diagram showing a transverse section of a wound bed surrounded by crypts where epithelial cells proliferate in and migrate from to cover the wound bed. ${ }^{24,29}$ (b) Immunofluorescence staining of wound beds in the colon of wild-type mice using antibody against $\beta$-catenin (green) and stained with actin (red) and DNA (blue). Mice were treated intrarectally with Lactobacillus rhamnosus GG (LGG) $\left(2.5 \times 10^{9} \mathrm{CFU}\right)$ or Hank's balanced salt solution (HBSS) for 2 days. The wound bed center is described in Methods and Supplementary Figure S1. Bar $=50 \mu \mathrm{m}$. (c) Endoscopic images of colonic

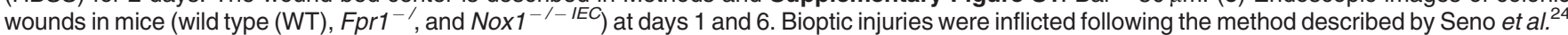
with $n=5$ mice per group. Mice were treated as above for 6 days. Dotted lines outline the depressed area margin of the lesion at each time point. (d) Graph shows percent wound closure on day 6 . Results are shown as means $\pm \mathrm{s}$.d. and ${ }^{*} P<0.05$, by Student's $t$-test. (e) Clinical disease activity index of mice subjected to dextran sodium sulfate (DSS) colitis for 7 days followed by recovery from colitis for 7 days. Graph is highlighted for intrarectal administration of LGG or HBSS for 3 days after DSS replacement with water; $n=6$ mice per group. Data in the graph are presented as mean \pm s.e.m. ${ }^{\star} P<0.05$, WT HBSS (blue) vs. WT LGG (red), and WT LGG vs. Fpr1 ${ }^{-/-}$LGG (violet) by analysis of variance (ANOVA) with Tukey's multiple comparison post-test.

epithelia by measuring cellular ROS using hydrocyanine 3, a stable intracellular ROS-sensitive fluorophore. ${ }^{21,25}$ Within 15 min of intrarectal administration of LGG suspension $\left(2.5 \times 10^{9} \mathrm{CFU}\right)$, but not HBSS control, WT mice showed rapid generation of ROS in the colonic epithelial cells (Supplementary Figure S2b and Figure 2b,c, white arrows), predominately in close proximity of the wounded area. Furthermore, administration of NAC in the mouse distal colon abrogated LGG-stimulated ROS generation (Figure 2b, c). Importantly, administration of Escherichia coli did not induce ROS generation, consistent with our past data indicating that specific strains of bacteria differ in their capacity to induce ROS generation. ${ }^{20}$ Minimal ROS generation was detected in IECs of $\mathrm{Fprl}^{-/-}$mice administered LGG (Figure 2b,c), implicating that this receptor is required for commensalmediated ROS generation. The NADPH oxidase family enzymes are the major sources of cellular ROS generation in non-phagocytic cells, ${ }^{26}$ with NOX1 abundantly expressed in
IECs along the crypt-luminal axis. ${ }^{27}$ ROS generation was markedly reduced in the IECs of Nox1 $1^{-/-I E C}$ but remained at WT levels in the Nox2 $2^{-1-}$ (the phagocyte NADPH oxidase) mouse treated with LGG. Finally, we found that the TLR signaling adaptor MyD88 was not necessary for LGG-stimulated ROS generation (Figure 2b, c). Taken together, these data show that recognition of the microbiota by FPR1 induced NOX1-dependent ROS generation within the enterocytes.

\section{Enteric commensal bacteria-stimulated FAK activation in murine colon depends on FPR1 and NOX1}

ROS modulate regulatory pathways important for wound healing $^{18}$ by reversible oxidative modification of redoxsensitive signaling intermediates, often enzymes bearing a low $\mathrm{pKa}$ "sensor" cysteines in their active sites, which thereby results in their catalytic inactivation. ${ }^{26,28}$ We recently demonstrated that enteric microbiota-induced ROS generation 


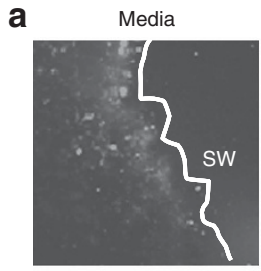

LGG, Boc2

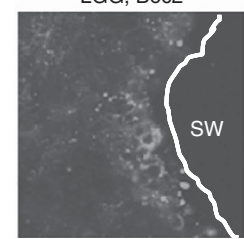

b

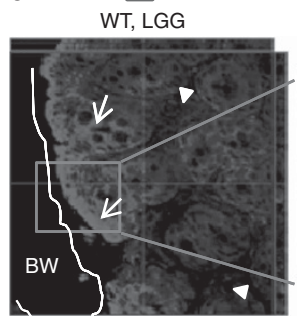

WT, E. coli

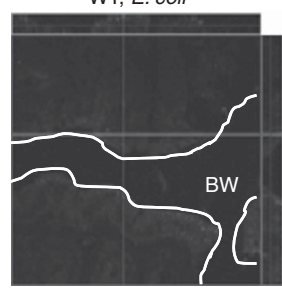

WT, NAC, LGG

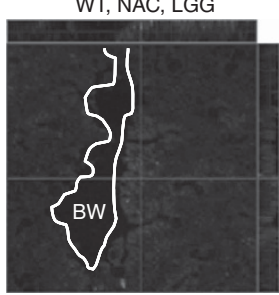

fMLF

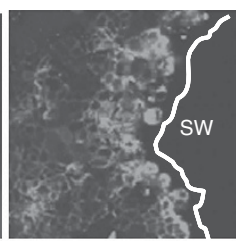

LGG, NAC

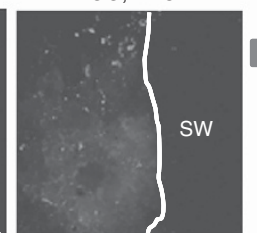

ROS

(CM-H2DCF-DA)

"SW" indicates scratch wound

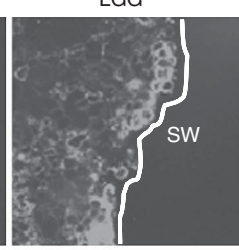
恋

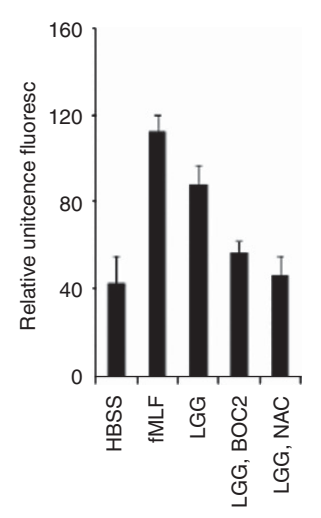

ROS (Hydrocyanine3) "BW" indicates biopsy wound

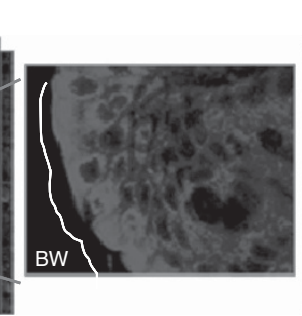

WT, HBSS
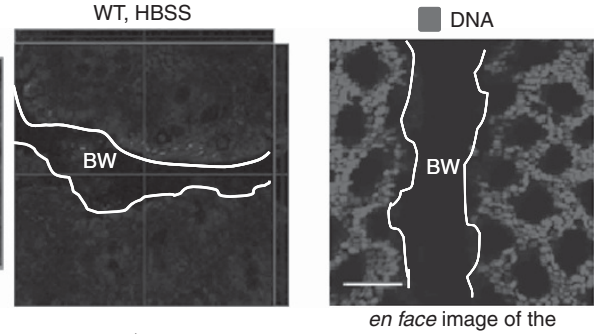

$\mathrm{Fpr1}^{-/-}$, LGG

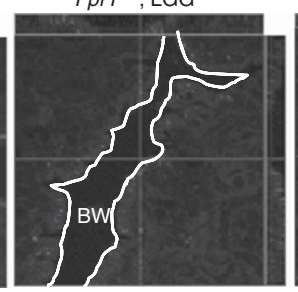

$M y D 88^{-/}$, LGG

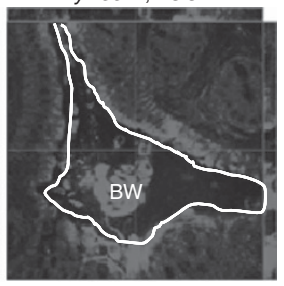

$N o \times 1^{-1-I E C}$, LGG

Nox2 ${ }^{-/}$, LGG
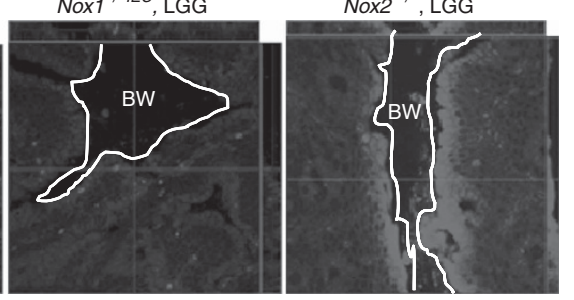

C

biopsy wound (BW)

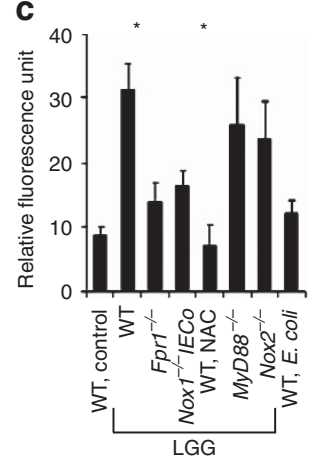

Figure 2 Commensal bacteria-induced reactive oxygen species (ROS) generation in intestinal epithelia requires formyl peptide receptor 1 (FPR1) and NADPH oxidase 1 (NOX1). (a) CM- $\mathrm{H}_{2}$ DCF-DA ( $5 \mu \mathrm{m}$ )-mediated detection of ROS in scratch-wounded (SW) polarized SK-CO15 cells treated with fMetLeu-Phe (fMLF) or Lactobacillus rhamnosus GG (LGG) $\left(2.5 \times 10^{7} \mathrm{CFU} \mathrm{ml}{ }^{-1}\right)$ over $15 \mathrm{~min}$. White lines depict SW area. Quantitative representation (right) of ROS production in panel a. Fluorescence intensity was measured by the ImageJ software and expressed in units of fluorescence. Results are shown as means \pm s.d. (b) Detection of ROS in vivo. Mice were loaded with hydrocyanine 3 followed by biopsy wounding (BW) of the epithelium. Mucosa was luminally treated for $15 \mathrm{~min}$ with Hank's balanced salt solution (HBSS) or LGG $\left(2.5 \times 10^{9} \mathrm{CFU}\right)$. Fluorescence was determined from en face wound bed by confocal laser scanning microscopy (Zeiss). Data represent three independent experiments with $n=3$ mice per group. White lines, white arrows, and white triangles depict biopsy wounds, enterocytes in colonic crypt units, and lamina propria, respectively. DNA was stained with To-pro 3 for tissue orientation. Bar $=50 \mu \mathrm{m}$. (c) Quantitative representation of ROS production in panel $\mathbf{b}$. Results are shown as means $\pm s$.d. and ${ }^{*} P<0.001$, by Student's $t$-test. Fluorescence intensity was measured by the ImageJ software and expressed in units of fluorescence. NAC, $N$-acetyl-cysteine.

inactivates LMW_PTP and SHP-2, two related redox-sensitive FAK phosphatases, and thereby activates FAK and paxillin phosphorylation in vitro; ${ }^{21}$ nonetheless, the receptor that recognized microbial signals and the cellular source that generated ROS remained unidentified. As these proteins are key regulators of epithelial cell migration, in this current study we investigated the role of FPR1 and NOX1 in FAK and paxillin phosphorylation in vivo (Figure 3). Within $15 \mathrm{~min}$ of the intrarectal administration of LGG suspension, but not HBSS, FAK was rapidly phosphorylated at tyrosine residue 861 (pFAK-Y861) over physiological steady-state conditions (control) as detected by immunoblotting ((Supplementary Figure S3a and Figure 3a) or immunofluorescence microscopy (Figure 3c, d) in WT mice. Phosphorylated FAK was located at the basal surface of the epithelial cells (Figure 3c,d). Importantly, phosphorylation of FAK 

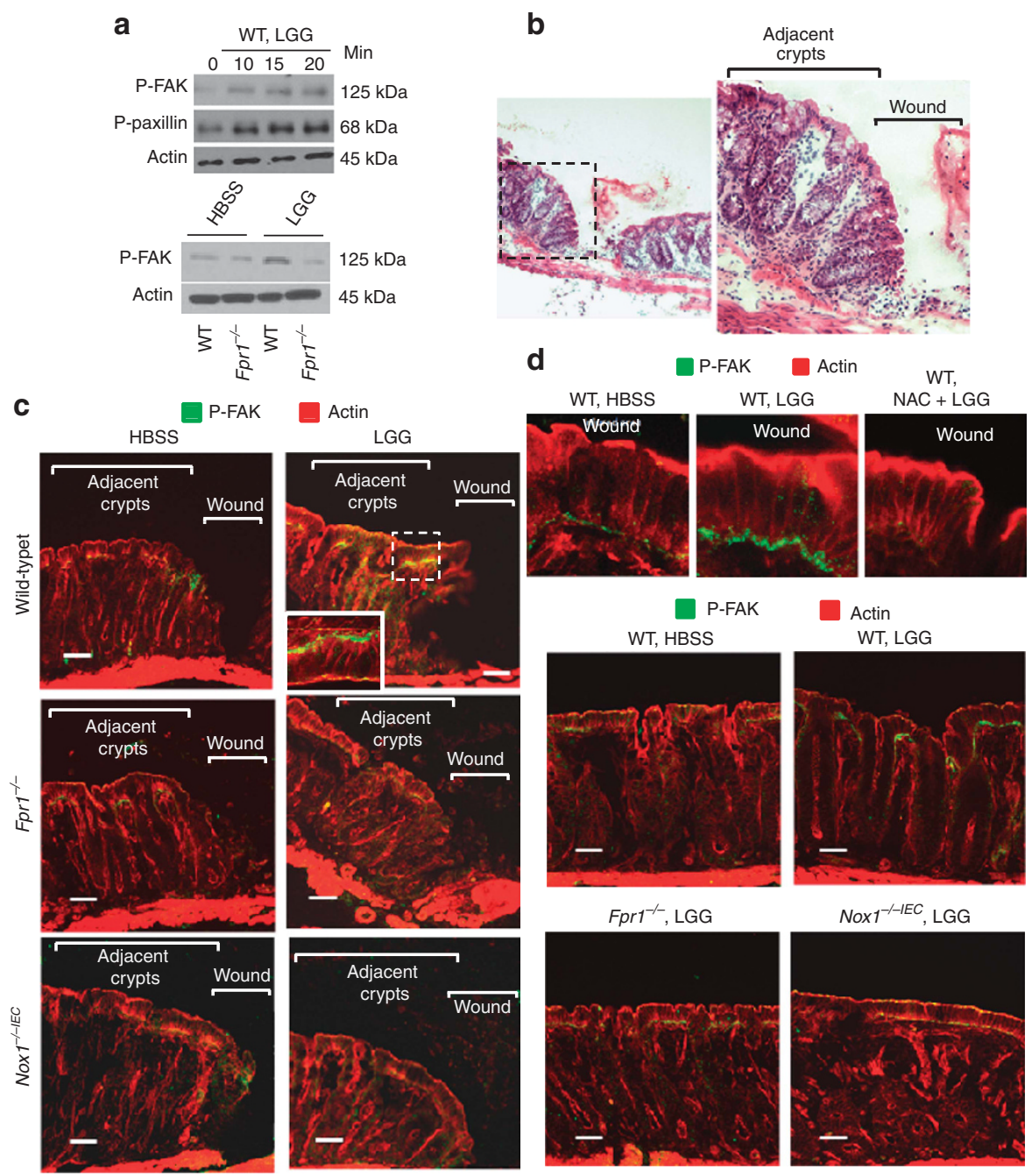

Figure 3 Enteric commensal bacteria-stimulated focal adhesion kinase (FAK) activation in murine colon depends on formyl peptide receptor 1 (FPR1) and NADPH oxidase 1 (NOX1). (a) Immunoblot analysis of phospho-FAK (P-FAK-Y861) in mouse colon at 15 min after biopsy injury and treatment with Lactobacillus rhamnosus GG (LGG) or Hank's balanced salt solution (HBSS). Data are representative of two independent experiments with $n=3$ mice per group. (b) Hematoxylin and eosin (H\&E) staining of a biopsy wound bed from wild-type (WT) mice 15 min after injury. (c-e) Immunofluorescence analysis of P-FAK in serial sections of colonic tissues harvested from mice treated intrarectally with LGG $\left(2.5 \times 10^{9} \mathrm{CFU}\right)$ or HBSS for 15 min. (c) Biopsy wounds ( $n=6$ lesions per group). White lines show crypts adjacent to the wound. (d) Biopsy wounds. $N$-acetyl-cysteine (NAC) was instilled 30 min before LGG treatment. (e) Intact colonic mucosa with $n=5$ per group. Bar $=50 \mu \mathrm{m}$. Data are representative of three independent experiments.

was markedly decreased in $\mathrm{Fprl}^{-/-}$and $\mathrm{Nox1}^{-/-I E C}$ mice (Figure 3a,c) and in WT mice pretreated with NAC (Figure 3d). Equal basal levels of unphosphorylated FAK were detected in WT, Fpr1 ${ }^{-1-}$, and Nox1 ${ }^{-1-I E C}$ mice (data not shown). Similarly, although much weaker, FPR1- and NOX1-dependent FAK activation was seen in unwounded mucosa (Figure 3e). Taken together, these data indicate that FPR1 and NOX1 are required for enteric commensal bacteriastimulated activation of FAK in IECs.

\section{Enteric commensal bacteria-stimulated epithelial cell migration in murine colon requires FPR1 and NOX1}

During restitution of colonic wounds, the epithelial cells migrate from the crypts adjacent to the wound site 24,29 (Supplementary Figure S3b, left panel). Figure 4a displays crypts adjacent to the wound bed at day 2 in the mouse distal colon. We measured the migration of enterocytes covering the wound bed by a pulse-chase experiment using EdU (5-ethynyl-2'-deoxyuridine) labeling of crypt epithelial cells following bioptic injury (Supplementary Figure S3b, right panel). EdU is a thymidine analog, which is incorporated in cells only at the $S$ phase. Once labeled, EdU-positive cells can be detected after several days. To determine the migration of enterocytes, colonic epithelial wounds were inflicted by biopsy forceps and EdU was injected intraperitonially. Wound bed tissues were subsequently harvested at different days after biopsy injury. Serial sections of wound beds were stained for EdU-positive cells (red) as well as immunostained for $\beta$-catenin to identify epithelial cells (green). Emigration of EdU-labeled enterocytes is seen (red) from the adjacent crypts one day after injury (Figure $4 \mathbf{b}$ ). In addition, after a 4-day chase period, 
a

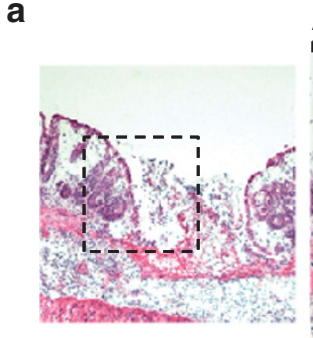

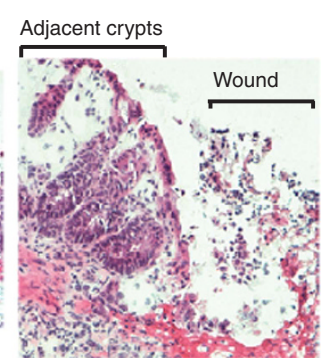

b

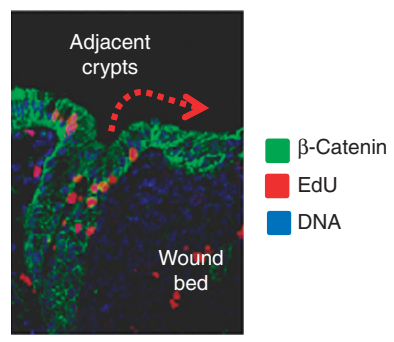

C $\square$-Catenin $\square \mathrm{EdU}$

DNA

Re-epithelialization: $\quad-=-=$ Partial

Complete
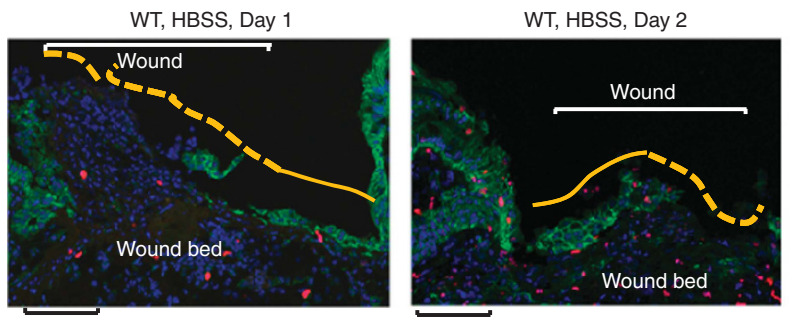

Adjacen
crypts

d

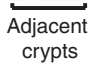

WT, HBSS, Day 2
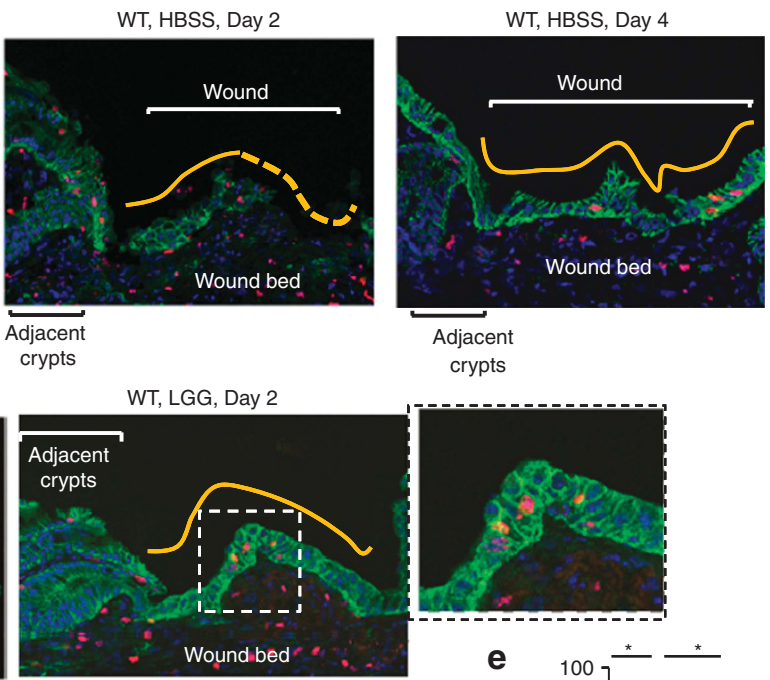

Adjacent

crypts
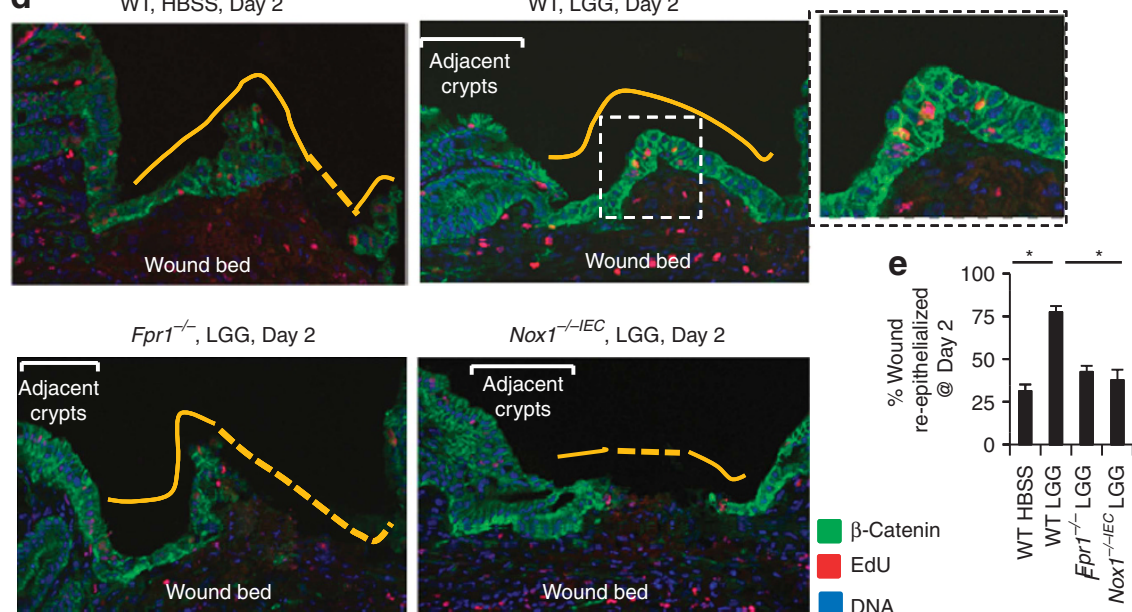

Figure 4 Enteric commensal bacteria-stimulated enterocyte migration in murine colon depends on formyl peptide receptor 1 (FPR1) and NADPH oxidase 1 (NOX1). (a) Hematoxylin and eosin (H\&E) staining of a biopsy wound bed from wild-type (WT) mice 2 days after injury. (b) EdU (5-ethynyl-2' deoxyuridine) -stained sections of a WT mouse 1 day after biopsy injury showing (EdU positive; red) epithelial cells emanating from a crypt. Mice were injected with EdU (intraperitoneally) immediately after endoscopic injury. Wound beds were harvested after 1 day and serial sections were stained for EdU-positive cells and $\beta$-catenin. (c) Re-epithelialization of wound beds with EdU-labeled enterocytes. Mice were treated as above for 1-4 days and injected with EdU ( intraperitoneally) immediately after endoscopy. Serial sections of wound beds were stained for EdU-positive cells and $\beta$-catenin. (d) Re-epithelialization of wound beds with EdU-labeled enterocytes in WT, Fpr1 ${ }^{-1-}$, and Nox $1^{-1-I E C}$ mice. Mice were treated as above with Hank's balanced salt solution (HBSS) or Lactobacillus rhamnosus GG (LGG) $\left(2.5 \times 10^{9} \mathrm{CFU}\right)$ for 2 days and injected with EdU immediately after endoscopy. Sections of wound beds were stained for EdU-positive cells and $\beta$-catenin with $n=5$ lesions per group. Data are representative of three independent experiments. White lines show crypts adjacent to the wound. Yellow lines indicate re-epithelialization where the solid yellow lines highlight complete and dotted lines highlight partial re-epithelialization. (e) Quantification of wound re-epithelialization on day 2. Results are shown as means \pm s.d. $(n=5)$ and ${ }^{*} P<0.05$ by Student's $t$-test.

we readily found EdU-positive cells in the monolayer of $\beta$-catenin containing epithelial cells completely covering the wound beds in WT control mice (Figure 4c). Interestingly, in the LGG-treated WT mice, wounds were covered with a monolayer of epithelial cells containing the EdU-marked cells within 2 days (Figure 4d,e). By comparison, the wound beds in the WT control or LGG-

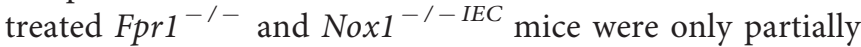
re-epithelialized at this time point (Figure 4d, e). A similar EdU labeling pulse-chase approach revealed that FPR1 is required in LGG-enhanced crypt-luminal migration of IECs in intact mucosa (Supplementary Figure S3c). Collectively, these data show that LGG-stimulated enterocyte migration is dependent on epithelial FPR1 and NOX1.

\section{Enteric commensal microbiota-stimulated ERK activation in murine colon requires FPR1 and NOX1}

Signaling pathways involved in cellular proliferation, including ERK mitogen-activated protein kinase pathways, are pivotal components of wound healing. We previously demonstrated that 

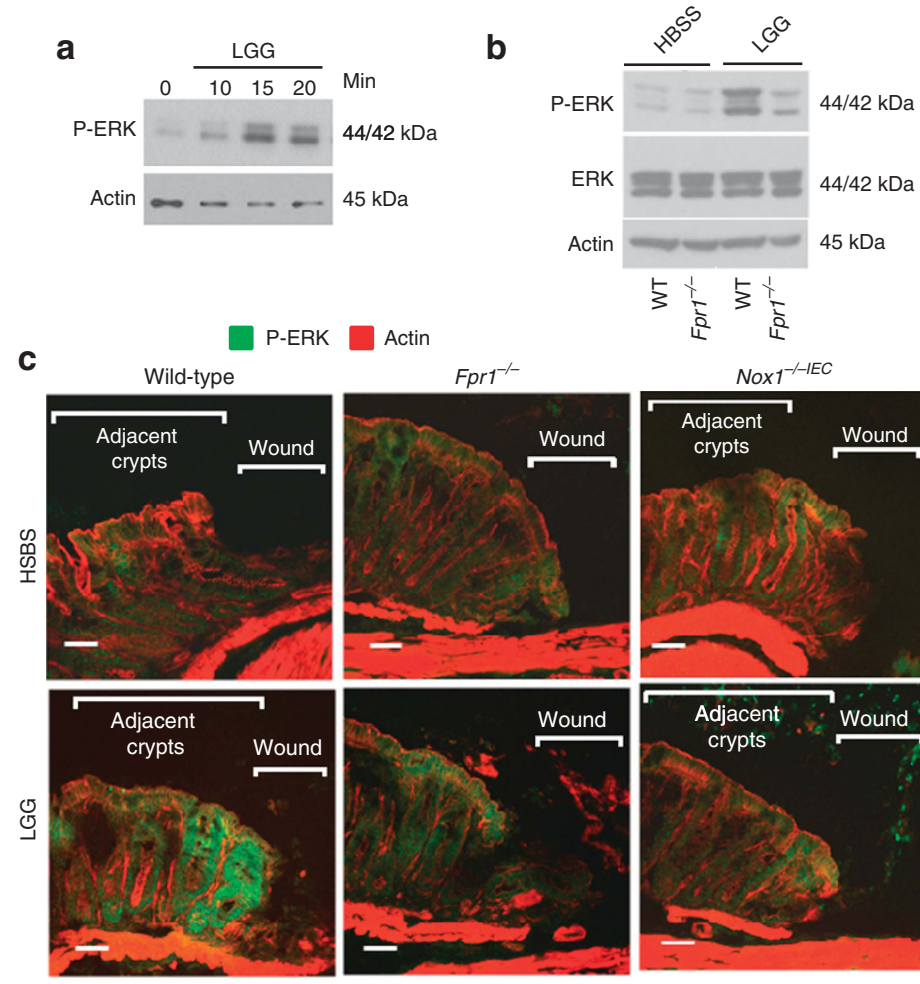

$$
\text { Noxi } 1^{-I I E C}
$$
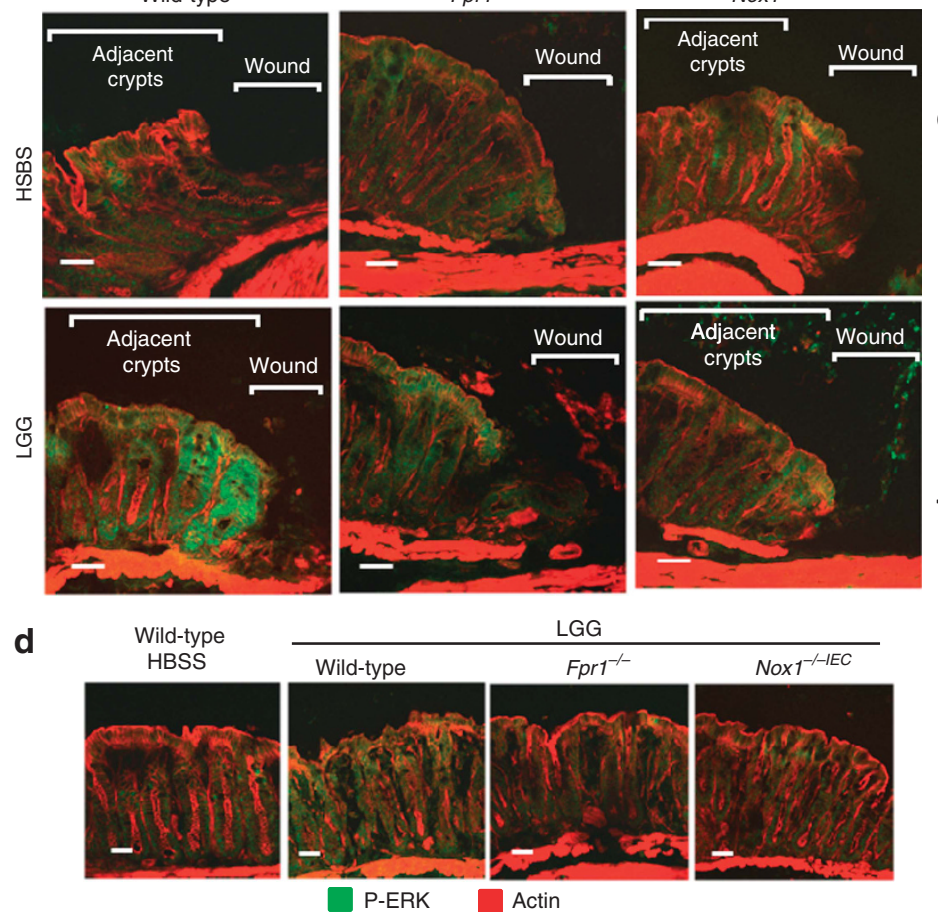

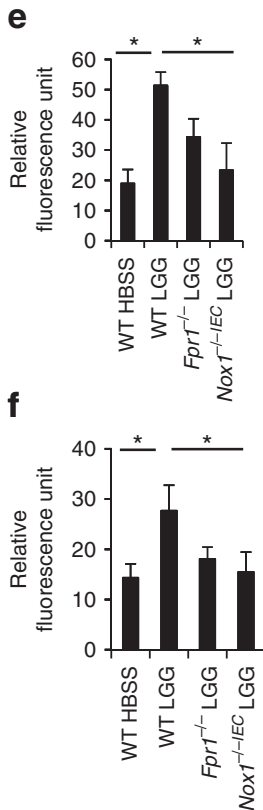

Figure 5 Commensal bacteria-stimulated ERK activation in crypts adjoining mucosal wounds requires formyl peptide receptor 1 (FPR1) and NADPH oxidase 1 (NOX1). (a) Immunoblot analysis for phospho-ERK (P-ERK) in colonic epithelial scrapings from harvested biopsy wounds located in the distal colon of wild-type and $\mathrm{Fpr1}^{-1-}$ mouse after intrarectal administration of Hank's balanced salt solution (HBSS) or Lactobacillus rhamnosus GG (LGG) for $15 \mathrm{~min}$. Data are representative of two independent experiments with $n=3$ mice per group. (b and $\mathbf{c})$ Immunofluorescence analysis of P-ERK in thin sections of colonic tissues from mice treated intrarectally with LGG $\left(2.5 \times 10^{9} \mathrm{CFU}\right)$ or HBSS for $15 \mathrm{~min}$. (b) Biopsy wounds with $n=7$ lesions per group. $\mathrm{Bar}=50 \mu \mathrm{m}$. (c) Intact colonic mucosa. (d and e) Quantitative representation of immunofluorescence analysis of P-ERK in panels $\mathbf{b}$ and $\mathbf{c}$. Results are shown as means \pm s.d. and ${ }^{*} P<0.05$ by Student's $t$-test. Data are representative of three independent experiments with $n=3$ mice per group.

commensal bacteria-induced ROS generation causes oxidative inactivation of the ERK phosphatase DUSP3 and increases ERK phosphorylation; ${ }^{30}$ however, sources of cellular ROS remained unidentified. In this current study, we thus assessed the role of FPR1 and NOX1 in ERK phosphorylation in vivo by inflicting biopsy injury in the mouse distal colon. Within $15 \mathrm{~min}$ of the intrarectal administration of LGG suspension, but not HBSS, ERK was rapidly phosphorylated in WT mice (Supplementary Figure S4 and Figure 5a,b). Interestingly, most phospho-ERK immunolocalized to the 4-5 crypt-villus units adjacent to the biopsy wounds (Figure 5b). Importantly, in response to LGG, markedly reduced levels of ERK phosphorylation was observed in $\mathrm{Fpr1}^{-1-}$ and Nox1 ${ }^{-1-I E C}$ mice and WT mice pretreated with NAC (Figure 5a,b,d and Supplementary Figure S4). Similarly, although much weaker, FPR1- and NOX1dependent ERK activation was observed in the unwound intact mucosa (Figure $5 c, e)$.

\section{FPR1 and NOX1 are required for enteric commensal bacteria-stimulated proliferation of wound-associated colonic epithelial cells}

Next, we examined IEC proliferation in crypts adjacent to the biopsy wound bed by EdU incorporation assay (Supplementary Figure S5a). Colonic epithelial wounds were inflicted by biopsy forceps and tissues were collected at different days after biopsy injury. EdU was injected intraperitoneally $2 \mathrm{~h}$ before harvesting the wound beds to label proliferating cells at the $S$ phase. As described before, ${ }^{24} 4-$ 5 crypts adjacent to wound bed showed increase in IEC proliferation (EdU positive; red) within 2 days of inflicting biopsy injury in WT control mice (Supplementary Figure S5b online and Figure 6a). To investigate the role of FPR1 and NOX1 in commensal microbiota-induced IEC proliferation, wounds were treated intrarectally with HBSS or LGG for 2 days and proliferation was assessed by EdU incorporation and 

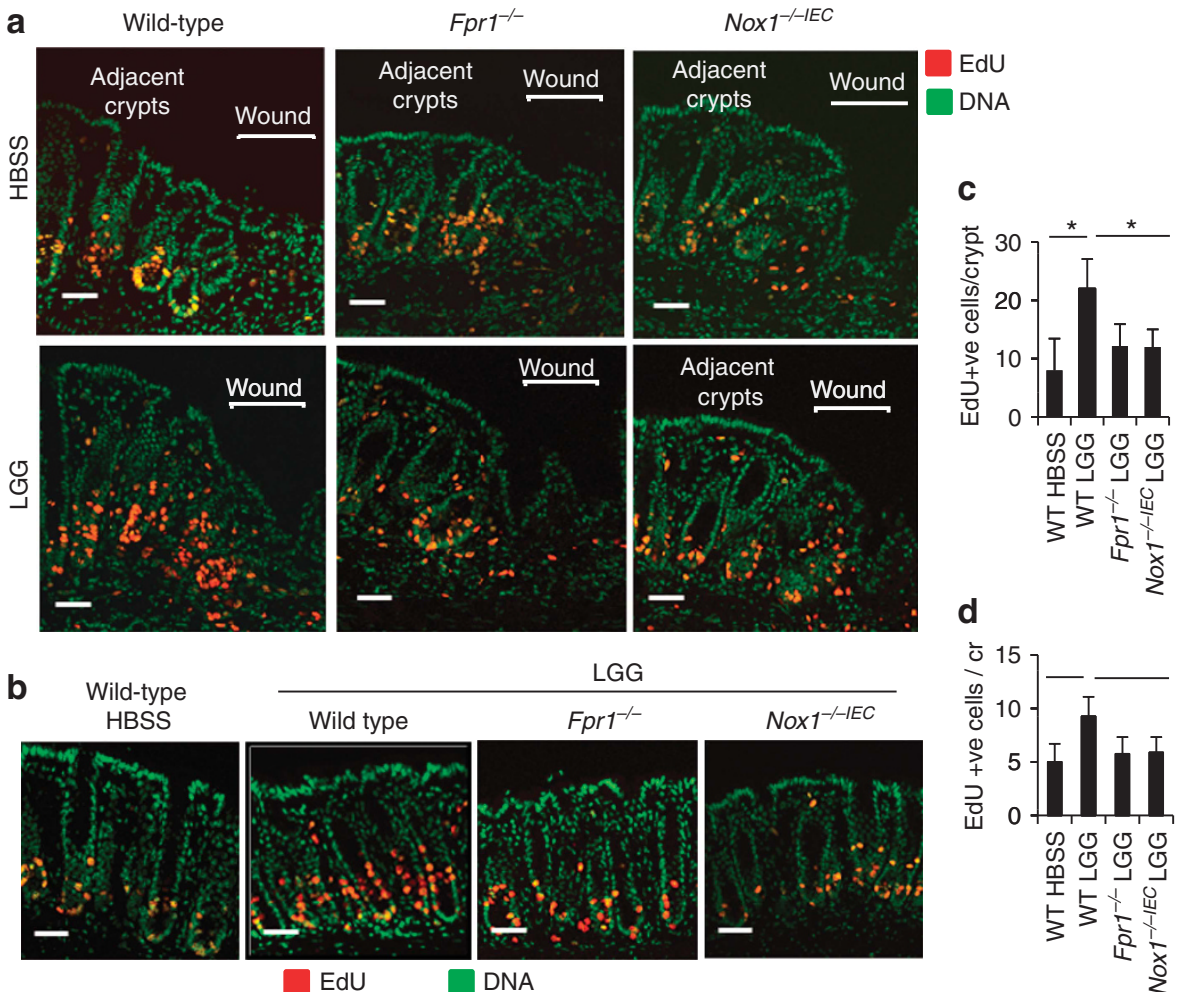

Figure 6 Commensal bacteria-stimulated enterocyte proliferation in crypts adjoining mucosal wounds requires formyl peptide receptor 1 (FPR1) and NADPH oxidase 1 (NOX1). (a and $\mathbf{b})$ Determination of enterocyte proliferation on day 2. Mice were treated intrarectally with Hank's balanced salt solution (HBSS) or Lactobacillus rhamnosus GG (LGG) for 2 days. EdU (5-ethynyl-2'-deoxyuridine) was injected intraperitoneally for a pulse of $2 \mathrm{~h}$ before harvesting of colonic tissues. Thin serial sections of colonic tissues were stained for EdU in cells at the S phase. (a) Biopsy wounds with $n=6$ lesions per group. White lines show crypts adjacent to the wound. (b) Intact colonic mucosa. Bar $=50 \mu \mathrm{m}$. Data are representative of three independent experiments with $n=3$ mice per group. (c and d) Quantification of EdU-positive cells. (c) Edu-positive cells in five crypts adjacent to the biopsy wounds were counted and expressed as Edu-positive cells per crypt. (d) Intact mucosa. Results are shown as means \pm s.d. $(n=6)$ and ${ }^{*} P<0.05$ by Student's $t$-test.

immunostaining with anti-Ki67 (Supplementary Figure S5c, d and Figure 6a,c). Treatment of wounds with LGG significantly increased the number of EdU-positive cells (red) in the 4-5 colonic crypts adjacent to wounds in WT mice. However, no significant increase in proliferation was detected in wound associated colonic crypts of Fpr1 ${ }^{-/-}$and Nox1 $1^{-/-I E C}$ mice (Figure 6a,c). Similarly, although much weaker, FPR1- and NOX1-dependent LGG-stimulated proliferative response was detected in the unwounded mucosa (Figure $\mathbf{6 b}, \mathbf{d}$ ). Taken together, these data indicate that FPR1 and NOX1 are required for enteric commensal bacteria-stimulated activation of ERK and induction of proliferation of IECs, cellular processes important for mucosal wound healing and homeostasis.

\section{DISCUSSION}

Repair of mucosal injury, termed restitution, requires induced and coordinated proliferation and migration of IECs. On the basis of our past demonstration of functional FPR on the apical surface of gut epithelia and our findings showing prorestitutive effects of FPR ligand AnxA1, we sought to investigate the role of FPR in host-commensal interactions that promote mucosal wound healing. FPRs represent an important class of pattern recognition receptor that can bind an array of exogenous bacterial peptides as well as endogenous mediators including AnxA1, lipoxin A4, and resolving D1. ${ }^{13,14,31}$ Neutrophils and monocytes perceive bacterial products via the FPRs, which elicit key innate immune processes in phagocytes, including onset of motility (chemotaxis), NOX2-catalyzed ROS generation, and regulation of signaling pathways. ${ }^{7}$

Although the role of FPRs in microbial perception and effector function in phagocytes goes back decades, only recently have they been detected in non-hematopoietic tissues including colonic and gastric epithelia, lung and nasal epithelia, as well as lens and retinal pigment epithelia, ${ }^{32,33}$ FPRs mediate migration of cultured IECs and enhance wound gap closure in response to bacterial formyl peptides. ${ }^{11,12}$ FPR ligands also promote wound closure in lung epithelial cells ${ }^{32}$ and human retinal pigment epithelial cell. ${ }^{33}$ Recently, FPR2-null mice have been reported to be involved in repair of chemically induced mucosal injury. ${ }^{15}$ In addition, during the resolution phase of inflammation, there is a temporal increase in proresolving mediators including resolvin D1 and lipoxin A4, which stimulate resolution via FPR2 receptor. ${ }^{14}$ Our laboratory previously demonstrated that the commensal microbiota enhance migration of IECs by modulating cell adhesion complexes, ${ }^{21}$ called focal adhesions (FAs) that stabilize migrating cells to the underlying matrix. One of the key regulatory proteins of FA complex is FAK, a cytoplasmic protein tyrosine kinase, which is under the regulation of ROS-sensitive phosphatases including PTPs, PTEN, and SHP2. ${ }^{17,34,35}$ We specifically demonstrated that 
enteric microbiota-induced ROS generation inactivates redoxsensitive tyrosine phosphatases toward FAK, and thereby upregulating its phosphorylation in vitro. ${ }^{21}$ By making endoscopic bioptic mucosal wounds in murine model, in this current report, we specifically show that commensal activate rapid phosphorylation of FAK and paxillin in an FPR1dependent manner. Importantly, these events were also

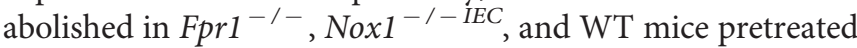
with NAC, strongly implicating involvement of FPR1dependent redox signaling. These present findings are consistent with our recent report that AnxA1, an endogenous FPR ligand, induces NOX1-dependent ROS generation via FPR1 leading to the oxidative inactivation of regulatory phosphatases including PTP-PEST and PTEN, resulting in the upregulation of FAK phosphorylation and subsequent wound gap closure. ${ }^{13}$

A number of studies implicated FPRs in epithelial cell proliferation, ${ }^{36,37}$ a critical component of wound restitution. Intriguingly, Helicobacter pylori $\mathrm{Hp}(2-20)$ peptide, an exogenous ligand derived from gastric epithelial microbiota, can also promote proliferation of gastric epithelial cells by interacting with FPRs; ${ }^{8}$ however, the molecular mechanism of microbiota-induced epithelial proliferation during wound repair via FPRs remained poorly understood. Cellular ROS generated from NOX enzymes mediate intracellular signaling important in wound repair and homeostasis, including cell cycle regulation, proliferation, and differentiation. ${ }^{18,26}$ Noxl is abundantly transcribed in IECs located both in the colonic crypts and luminal tips. ${ }^{27}$ Recently, Coant et al. ${ }^{38}$ showed that mice with a germline deletion in Nox1 demonstrated defective intestinal epithelial differentiation. In this current study, our findings specifically established that microbiota-induced ERK phosphorylation and subsequent IEC proliferation in woundassociated enterocytes were dependent on ROS generation mediated by FPR1 and NOX1.

The mechanisms by which the host perceives prokaryotic contact and transduces this into physiological, rather than pathological, results are largely unknown. We identify a central role of FPR1 and NOX1 in mediating the commensal microbiota-stimulated proproliferative and promigratory effects on enterocytes that facilitate mucosal repair and homeostasis (Figure 7). Identification of the optimal commensal species and/or microbial products as well as indigenous host molecules that modulate the physiological ROS generation through epithelial FPR1-NOX1 signaling pathways will be crucial for developing future therapeutic approaches for a range of mucosal disorders.

\section{METHODS}

Mouse models. All animal experiments were approved by the Institutional Animal Care and Use Committee at Emory University (Atlanta, GA) and performed according to the National Institutes of Health guidelines. The mice had ad libitum access to a standard diet and water until reaching the desired age and/or weight. Animals were maintained on a 12-h light/12-h dark cycle under pathogen-free
Formyl peptide / Commensal bacteria

L. rhamnosus GG (LGG)
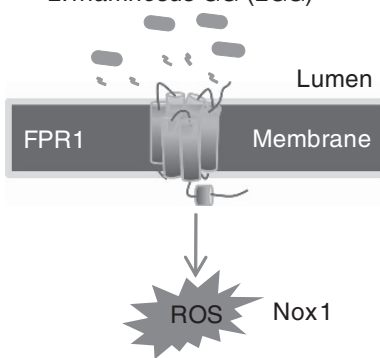

Signal transduction

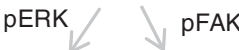

Cell proliferation Cell migration

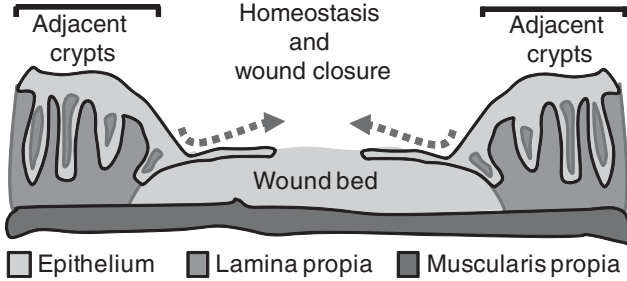

Figure 7 Model depicts the effects of commensal bacteria on restitution of colonic epithelial wounds. Formyl peptide receptor 1 (FPR1) receptors are expressed on the apical surface of colonic enterocytes. The FPR1 receptor perceives signals from commensal bacteria to elicit reactive oxygen species (ROS) via activation of NOX1. ROS mediates activation/ phosphorylation of ERK (P-ERK) and focal adhesion kinase (P-FAK) with resultant effects on proliferation and also turnover of focal adhesions. Overall enhancement of cell proliferation and migration promotes wound closure and contributes to overall homeostasis.

conditions. Fpr1 ${ }^{-/-}$mouse were purchased from Taconic (Germantown, NY). Colonies of $\mathrm{Fprl}^{-/-}, \mathrm{Nox1}^{-/-I E C}$, and MyD88 ${ }^{-/-}$mice were established and maintained in our research facility. Nox $1^{-/-I E C}$ mice were described by Leoni et al. ${ }^{24}$ Nox2-null mice were purchased from the Jackson Laboratory (Bar Harbor, ME). Genotyping was accomplished by PCR of the WT mFprl allele or mutated gene carrying the neomycin resistance gene $\left(\right.$ neo $\left.^{\mathrm{r}}\right)$.

Endoscope used in live mice. To create discrete mucosal injuries in the mouse colon and to monitor their regeneration, we used a high-resolution miniaturized colonoscope system (Coloview Veterinary Endoscope; Karl Stortz, Goleta, CA). This system consisted of a miniature rigid endoscope $(1.9-\mathrm{mm}$ outer diameter), a xenon light source, a triple chip high-resolution CCD camera, and an operating sheath with instrument channels and an air/water injection bulb to regulate inflation of the mouse colon (all from Karl Storz). The endoscope with outer operating sheath was inserted into the mid-descending colon and the mucosa was surveyed to the anorectal junction. Then, the flexible biopsy forceps with a diameter of 3 French were inserted to remove single full-thickness areas of the entire mucosa and submucosa. Endoscopic procedures were viewed with high resolution $(1,024 \times 768$ pixels $)$ images on a flat panel 
color monitor. This enabled us to take both video and still images of the endoscopic procedures. ${ }^{24}$ The Image J software (National Institutes of Health, Bethesda, MD) was used to analyze the wound sizes. The night before the initial biopsy injury, food was removed from the mouse cages. The following morning, mice were anesthetized using ketamine and xylazine. We took particular care to avoid penetration of the muscularis propria.

Wound bed tissue preparation. Wound bed gross morphology was studied with a dissection microscope. Mucosal tissues with the wound bed were preserved in OCT media and frozen to perform immunofluorescence microscopy and histopathologic analysis. For histological analysis, wounds were oriented and cut in a proximal to distal manner and sections were prepared in $7-\mu \mathrm{m}$ increments. ${ }^{24,39}$ The sections with the largest area of the wound bed (highlighted by dashed lines in Supplementary Figure S1a and with asterisk in Supplementary Figure S1b) were considered the center of the wound ${ }^{24,39}$ and used for immunofluorescence staining.

Cell migration in vivo. To assess for epithelial migration to cover a wound bed, mice were injected intraperitoneally with EdU (100 $\mu \mathrm{g} \mathrm{g}^{-1}$ body weight) at the time of biopsy injury and were euthanized 2-4 days later (chase: 2-4 days). Immunohistochemical localization of EdU-labeled cells were carried out by Click-iT EdU Alexa Fluor Imaging Kit (Life Technologies, Carlsbad, CA). Nuclei were stained with the To-pro 3 (Molecular Probes, Eugene, OR). Sections were also immunostained for $\beta$-catenin to identify enterocytes.

To assess for epithelial migration in intact colonic tissue, mice were injected intraperitoneally with EdU. Migration of enterocytes was measured by determining locations of the foremost EdU-labeled enterocytes' colonic crypt and was recorded as the percent of epithelial cell positions along the crypt-luminal axis of well-oriented crypt units.

Cell proliferation in vivo. After inflicting biopsy injury in mice, EdU was administered intraperitoneally at different time points (days 0, 1, and 2). Mice were killed $2 \mathrm{~h}$ after EdU administration (pulse: $2 \mathrm{~h}$ ); tissue sections containing wound beds were collected in OCT medium and were frozen. S-phase cells were detected with procedure described above. Proliferating cells were also determined by immunofluorescence using anti-Ki67 antibody.

Immunoblotting and immunofluorescence. To collect epithelial cells, intrarectal administrations of HBSS (Hank's buffer), LGG, or fMLF for the indicated times were performed in anesthetized mice. Mice were euthanized, colons were opened along the mesenteric border, epithelial tissues were scrapped and lysed in radioimmunoprecipitation assay buffer $(100 \mathrm{mg}$ tissue per $\mathrm{ml}$ of buffer) followed by centrifugation at 16,000 r.p.m. for $20 \mathrm{~min}$ at $4{ }^{\circ} \mathrm{C}$. Antibodies were obtained as follows: anti-ERK, phospho-ERK (Cell Signaling, Danvers, MA), $\beta$-actin (SigmaAldrich Corp., St Louis, MO), phospho-FAK (Sigma-Aldrich Corp.), phospho-paxillin (Abcam, Cambridge, MA), fluorescein isothiocyanate-conjugated goat anti-rabbit $\operatorname{IgG}$ (Jackson ImmunoResearch, West Grove, PA), and horseradish peroxidase- conjugated donkey anti-rabbit or sheep anti-mouse secondary antibody (GE Healthcare, Little Chalfont, Buckinghamshire, UK). Immunoblot and immunofluorescent labeling slips were performed as described previously. ${ }^{13}$ Nuclei were stained with To-Pro-3 iodide (Molecular Probes). Fluorescent images were acquired by laser confocal microscopy.

ROS assay in vivo. For detection of ROS in the murine biopsy injury model, a nontoxic ROS-sensitive dye hydrocyanine 3 was used. ${ }^{25}$ Mice were anesthetized, followed by intrarectal injection with nontoxic ROS-sensitive dye hydrocyanine 3 that fluoresces when oxidized. After $30 \mathrm{~min}$ of Hydro-Cy3 injection, biopsy injuries were made, and administration of HBSS, LGG (2.5 × $10^{9} \mathrm{CFU}$ ), or fMLF for different time points were performed. Mice were killed, the colons were opened along the mesenteric border, and tissues were collected. After mounting on slides, samples were examined by confocal laser scanning microscopy. Images were captured using $535 \mathrm{~nm}$ laser extinction and $560 \mathrm{~nm}$ emission filter. ImageJ software (National Institutes of Health) was used to analyze fluorescence intensity.

\section{ROS assay in vitro}

Cell Culture. Human IEC line SK-CO15 was grown on membrane inserts in high glucose $\left(4.5 \mathrm{gl}^{-1}\right)$ Dulbecco's modified Eagle's medium supplemented with $10 \%$ fetal bovine serum, $100 \mathrm{U} \mathrm{ml}^{-1}$ penicillin, $100 \mu \mathrm{g} \mathrm{ml}^{-1}$ streptomycin, 15 mM HEPES (pH 7.4), 2 mM L-glutamine, and 1\% nonessential amino acids at $37^{\circ} \mathrm{C}$ in a $4 \% \mathrm{CO}_{2}$ incubator.

ROS detection. Scratch wounds were performed with plastic micropipette tips on polarized SK-CO15 cells grown on membrane inserts. Epithelial cells were treated with LGG $\left(5 \times 10^{7} \mathrm{CFU} \mathrm{ml}^{-1}\right)$ (ATCC, Manassas, VA; 53103) or fMLF (500 nM) (Sigma-Aldrich) for the indicated times were washed with HBSS and incubated in the dark with CM- $\mathrm{H}_{2} \mathrm{DCF}-\mathrm{DA}$ (Molecular Probes) for an additional $5 \mathrm{~min}$ as described previously by Wentworth et al. ${ }^{30}$ All images were acquired using a confocal laser scanning microscope (Zeiss LSM 510, Jena, Germany) at $\times 40$ magnification. Images were captured using $488 \mathrm{~nm}$ laser for excitation and a 515-540 nм emission filter. Quantification of fluorescence intensity was determined using ImageJ (National Institutes of Health, Bethesda, MD).

Induction of colitis. Four percent ( $\mathrm{wt} \mathrm{vol}^{-1}$ ) DSS (molecular mass, $36-50 \mathrm{kDa}$ ) was dissolved in purified water and orally administered to mice as described previously. ${ }^{13,21}$ Mice were allowed free access to food and water containing 4\% DSS from day 0 until day 7 and DSS was withdrawn to allow recovery from colitis for additional 4 days. Mice were administered with intrarectal injections of LGG suspension in HBSS buffer for additional 3 days. Mice were killed on day 14. Daily clinical assessment of DSS-treated animals included evaluation of stool consistency, detection of blood in stool, and body weight loss measurements. An individual score (ranging from 0 to 4) was attributed for each one of these parameters, and a disease activity index ranging from 0 to 4 was calculated by combining all three scores. 
Statistics. Quantitative data are expressed as mean \pm s.e.m. or s.d. for each treatment group. Statistical comparisons were performed by two-tailed Student's $t$-test or analysis of variance with Tukey's multiple comparison post-test (GraphPad Prism; GraphPad Software, La Jolla, CA). P-values $<0.05$ were considered significant.

SUPPLEMENTARY MATERIAL is linked to the online version of the paper at http://www.nature.com/mi

\section{ACKNOWLEDGMENTS}

We thank Dr Charles Parkos for critically reviewing the manuscript. This work was supported by grants R01DK089763, RO1DK055679 (to A.S.N.), R01Al64462 (to A.N.), and the Emory Digestive Diseases Research Development Center Core Grant (DK 064399). A.A. is supported by a Research Fellowship from Crohn's and Colitis Foundation of America.

\section{DISCLOSURE}

The authors declared no conflict of interest.

c 2014 Society for Mucosal Immunology

\section{REFERENCES}

1. Loftus, E.V. Jr. Clinical epidemiology of inflammatory bowel disease Incidence, prevalence, and environmental influences. Gastroenterology 126, 1504-1517 (2004).

2. Bewtra, M, Su, C \& Lewis, J.D. Trends in hospitalization rates for inflammatory bowel disease in the United States. Clin. Gastroenterol. Hepatol. 5, 597-601 (2007).

3. Pull, S.L., Doherty, J.M., Mills, J.C., Gordon, J.I. \& Stappenbeck, T.S. Activated macrophages are an adaptive element of the colonic epithelial progenitor niche necessary for regenerative responses to injury. Proc. Natl. Acad. Sci. 102, 99-104 (2005).

4. Neish, A.S. Microbes in gastrointestinal health and disease. Gastroenterology 136, 65-80 (2009).

5. Hooper, L.V. et al. Molecular analysis of commensal host-microbial relationships in the intestine. Science 291, 881-884 (2001).

6. Rakoff-Nahoum, S, Paglino, J, Eslami-Varzaneh, F, Edberg, S \& Medzhitov, R. Recognition of commensal microflora by toll-like receptors is required for intestinal homeostasis. Cell 118, 229-241 (2004).

7. Ye, R.D. et al. International Union of Basic and Clinical Pharmacology. LXXIII. Nomenclature for the formyl peptide receptor (FPR) family. Pharmacol. Rev. 61, 119-161 (2009).

8. de Paulis, A. et al. Helicobacter pylori $\mathrm{Hp}(2-20)$ promotes migration and proliferation of gastric epithelial cells by interacting with formyl peptide receptors in vitro and accelerates gastric mucosal healing in vivo. J. Immunol. 183, 3761-3769 (2009).

9. Southgate, E.L. et al. Identification of formyl peptides from Listeria monocytogenes and Staphylococcus aureus as potent chemoattractants for mouse neutrophils. J. Immunol. 181, 1429-1437 (2008).

10. Bylund, J, Samuelsson, M, Collins, L.V. \& Karlsson, A. NADPH-oxidase activation in murine neutrophils via formyl peptide receptors. Exp. Cell Res. 282, 70-77 (2003).

11. Babbin, B.A. et al. Annexin I regulates SKCO-15 cell invasion by signaling through formyl peptide receptors. J. Biol. Chem. 281, 19588-19599 (2006).

12. Babbin, B.A. et al. Formyl peptide receptor-1 activation enhances intestinal epithelial cell restitution through phosphatidylinositol 3-kinase-dependent activation of Rac1 and Cdc42. J. Immunol. 179, 8112-8121 (2007).

13. Leoni, G. et al. Annexin A1, formyl peptide receptor, and NOX1 orchestrate epithelial repair. J. Clin. Invest. 123, 443-454 (2013).

14. Norling, L.V., Dalli, J, Flower, R.J., Serhan, C.N. \& Perretti, M. Resolvin D1 limits polymorphonuclear leukocyte recruitment to inflammatory loci: receptor-dependent actions. Arterioscler. Thromb. Vasc. Biol. 32, 19701978 (2012).

15. Chen, K. et al. Formylpeptide receptor-2 contributes to colonic epithelial homeostasis, inflammation, and tumorigenesis. J. Clin. Invest. 123, 1694-1704 (2013)
16. Tsukagoshi, H, Busch, W \& Benfey, P.N. Transcriptional regulation of ROS controls transition from proliferation to differentiation in the root. Cell 143, 606-616 (2010).

17. Chiarugi, P. et al. Reactive oxygen species as essential mediators of cell adhesion: the oxidative inhibition of a FAK tyrosine phosphatase is required for cell adhesion. J. Cell Biol. 161, 933-944 (2003).

18. Love, N.R. et al. Amputation-induced reactive oxygen species are required for successful Xenopus tadpole tail regeneration. Nat. Cell Biol. 15, 222-228 (2012).

19. Wentworth, C.C., Jones, R.M., Kwon, Y.M., Nusrat, A \& Neish, A.S. Commensal-epithelial signaling mediated via formyl peptide receptors. Am. J. Pathol. 177, 2782-2790 (2010).

20. Kumar, A. et al. Commensal bacteria modulate cullin-dependent signaling via generation of reactive oxygen species. EMBO J. 26, 4457-4466 (2007).

21. Swanson, P.A. 2nd et al. Enteric commensal bacteria potentiate epithelial restitution via reactive oxygen species-mediated inactivation of focal adhesion kinase phosphatases. Proc. Natl Acad. Sci. USA 108 8803-8808 (2011).

22. Sieg, D.J., Hauck, C.R. \& Schlaepfer, D.D. Required role of focal adhesion kinase (FAK) for integrin-stimulated cell migration. J. Cell Sci. 112 (Part 16), 2677-2691 (1999).

23. Owen, K.A., Abshire, M.Y., Tilghman, R.W., Casanova, J.E. \& Bouton, A.H. FAK regulates intestinal epithelial cell survival and proliferation during mucosal wound healing. PLoS One 6, e23123 (2011).

24. Seno, H. et al. Efficient colonic mucosal wound repair requires Trem2 signaling. Proc. Natl. Acad. Sci. USA 106, 256-261 (2009).

25. Kundu, K. et al. Hydrocyanines: a class of fluorescent sensors that can image reactive oxygen species in cell culture, tissue, and in vivo. Angew. Chem. Int. Ed. Engl.48, 299-303 (2009).

26. Aguirre, J \& Lambeth, J.D. Nox enzymes from fungus to fly to fish and what they tell us about Nox function in mammals. Free Radic. Biol. Med. 49, 1342-1353 (2010).

27. Laurent, E. et al. Nox1 is over-expressed in human colon cancers and correlates with activating mutations in K-Ras. Int. J. Cancer 123, 100-107 (2008).

28. Hurd, T.R., DeGennaro, M \& Lehmann, R. Redox regulation of cell migration and adhesion. Trends Cell. Biol. 22, 107-115 (2012).

29. Miyoshi, H, Ajima, R, Luo, C.T., Yamaguchi, T.P. \& Stappenbeck, T.S. Wnt5a potentiates TGF-beta signaling to promote colonic crypt regeneration after tissue injury. Science 338, 108-113 (2012).

30. Wentworth, C.C., Alam, A, Jones, R.M., Nusrat, A \& Neish, A.S. Enteric commensal bacteria induce extracellular signal-regulated kinase pathway signaling via formyl peptide receptor-dependent redox modulation of dual specific phosphatase 3. J. Biol. Chem. 286, 38448-38455 (2011).

31. Gronert, K. et al. A role for the mouse 12/15-lipoxygenase pathway in promoting epithelial wound healing and host defense. J. Biol. Chem. 280, 15267-15278 (2005).

32. Shao, G. et al. Formyl peptide receptor ligands promote wound closure in lung epithelial cells. Am. J. Respir. Cell. Mol. Biol. 44, 264-269 (2011).

33. Zhang, X.G. et al. Activation of formyl peptide receptor-1 enhances restitution of human retinal pigment epithelial cell monolayer under electric fields. Invest. Ophthalmol. Vis. Sci. 52, 3160-3165 (2011).

34. Mitra, S.K., Hanson, D.A. \& Schlaepfer, D.D. Focal adhesion kinase: in command and control of cell motility. Nat. Rev. Mol. Cell. Biol. 6, 56-68 (2005).

35. Shao, D. et al. Redox modification of cell signaling in the cardiovascular system. J. Mol. Cell. Cardiol. 52, 550-558 (2012).

36. Khau, T. et al. Annexin-1 signals mitogen-stimulated breast tumor cell proliferation by activation of the formyl peptide receptors (FPRs) 1 and 2 . FASEB J. 25, 483-496 (2011).

37. Lee, S.Y. et al. F2L, a peptide derived from heme-binding protein, inhibits LL-37-induced cell proliferation and tube formation in human umbilical vein endothelial cells. FEBS Lett. 582, 273-278 (2008).

38. Coant, N. et al. NADPH oxidase 1 modulates WNT and NOTCH1 signaling to control the fate of proliferative progenitor cells in the colon. Mol. Cell. Biol. 30, 2636-2650 (2010)

39. Manieri, N.A., Drylewicz, M.R., Miyoshi, H \& Stappenbeck, T.S. Igf2bp1 is required for full induction of Ptgs2 mRNA in colonic mesenchymal stem cells in mice. Gastroenterology 143, 110-121 e110 (2012). 